\title{
Affine geometry of equal-volume polygons in 3-space
}

\author{
Marcos Craizer and Sinesio Pesco
}

\begin{abstract}
Equal-volume polygons are obtained from adequate discretizations of curves in 3-space, contained or not in surfaces. In this paper we explore the similarities of these polygons with the affine arc-length parameterized smooth curves to develop a theory of discrete affine invariants. Besides obtaining discrete affine invariants, equal-volume polygons can also be used to estimate projective invariants of a planar curve. This theory has many potential applications, among them evaluation of the quality and computation of affine invariants of silhouette curves.
\end{abstract}

Mathematics Subject Classification (2010). 53A15, 53A20.

Keywords. Darboux vector field, Affine arc-length parameterization, Affine evolute, Projective length, Discrete affine geometry.

\section{Introduction}

We say that a smooth curve $\gamma(t)$ in 2-space is parameterized by affine arclength if $\left[\gamma^{\prime}(t), \gamma^{\prime \prime}(t)\right]=1$, where $[\cdot, \cdot]$ denotes the determinant of 2 vectors. For polygons, the corresponding condition is that the area of the triangle determined by three consecutive vertices is constant. Planar polygons satisfying this last condition are called equal-area and the affine geometry of these polygons has been recently studied ([2, [11]). In this paper we generalize this study to polygons in 3 -space by considering the concept of equal-volume polygons. Since we obtain discrete counterparts of known objects of the smooth theory, our results clearly belong to the field of Discrete Differential Geometry.

For a smooth curve $\phi$ contained in a surface $M$, we say that the parameterization $\phi(t)$ is adapted to $M$ if

$$
\left[\phi^{\prime}(t), \phi^{\prime \prime}(t), \xi(t)\right]=1,
$$

The first author thanks CNPq for financial support during the preparation of this paper. 
where $[\cdot, \cdot, \cdot]$ denotes the determinant of 3 vectors and $\xi$ is the parallel Darboux vector field of $\phi \subset M([3)$. In centro-affine geometry, we consider curves $\phi$ in 3-space together with a distinguished origin $O$, and we say that $\phi(t)$ is parameterized by centro-affine arc-length with respect to $O$ if

$$
\left[\phi(t)-O, \phi^{\prime}(t), \phi^{\prime \prime}(t)\right]=1,
$$

([6]). A smooth curve $\Phi(t)$ in 3-space is parameterized by affine arc-length if

$$
\left[\Phi^{\prime}(t), \Phi^{\prime \prime}(t), \Phi^{\prime \prime \prime}(t)\right]=1,
$$

$([5,[9])$. We observe that, in all these contexts, the basic condition is the constancy of some volume. In this paper, we describe polygons in 3-space whose corresponding volumes are constant, and we call them equal-volume polygons. We obtain affine invariant measures only for these equal-volume polygons, but we also describe a simple algorithm that, by re-sampling an arbitrary polygon, obtain an equal-volume one.

We say that a smooth curve $\phi$ contained in a surface $M$ is non-degenerate if its osculating plane does not coincide with the tangent plane of $M$ at any point. For such curves, there exists a vector field $\xi$ tangent to $M$ and transversal to $\phi$ such that its derivative $\xi^{\prime}$ is tangent to $M$. The direction defined by $\xi$ is unique and is called the Darboux direction of $\phi \subset M$. Moreover, there exists a vector field $\xi$ in the Darboux direction such that $\xi^{\prime}$ is tangent to the curve $\phi$, i.e.,

$$
\xi^{\prime}(t)=-\sigma(t) \phi^{\prime}(t),
$$

for some scalar function $\sigma$. This vector field is unique up to a multiplicative constant and is called the parallel Darboux vector field. It turns out that $\phi \subset M$ is a silhouette curve with respect to some point $O$ if and only if $\sigma$ is constant ( $\underline{3})$.

As a discrete model for curves contained in surfaces, consider a polyhedron $M$ whose faces are planar quadrilaterals and let $\phi(i)$ be vertices of a polygon $\phi$ whose sides are connecting opposite edges of a face of $M$. The edges of $M$ containing vertices $\phi(i)$ correspond to Darboux directions and we can choose a vector field $\xi(i)$ in this direction such that the difference $\xi(i+1)-\xi(i)=$ $\xi^{\prime}\left(i+\frac{1}{2}\right)$ is parallel to the corresponding side of the polygon $\phi$. This vector field is unique up to a multiplicative constant, and is called the parallel Darboux vector field. We can write a discrete counterpart of equation (1.4), namely

$$
\xi^{\prime}\left(i+\frac{1}{2}\right)=-\sigma\left(i+\frac{1}{2}\right) \phi^{\prime}\left(i+\frac{1}{2}\right),
$$

for some scalar function $\sigma$, where we are replacing derivatives by differences. We prove that $\phi$ is a silhouette polygon for the polyhedron $M$ if and only if $\sigma$ is constant. This result may be used as a measure of quality of a silhouette polygon.

A non-degenerate curve $\phi \subset M$ admits a parameterization satisfying equation (1.1), unique up to a translation. The plane $\mathcal{A}=\mathcal{A}(t)$ generated by $\left\{\phi(t), \phi^{\prime \prime}(t)\right\}$ is called the affine normal plane, while the envelope $\mathcal{B}$ of these affine normal planes is a developable surface $\mathcal{B}$ called the affine focal set of 
the pair $\phi \subset M([3,4])$. For silhouette curves relative to $O$, equation (1.1) reduces to equation 1.2 .

We say that the polygon $\phi$ contained in the polyhedron $M$ is equal-volume if

$$
\left[\phi^{\prime}\left(i-\frac{1}{2}\right), \phi^{\prime}\left(i+\frac{1}{2}\right), \xi(i)\right]=1,
$$

for all $i$. Note that equation 1.6 is a discrete counterpart of equation (1.1). For such polygons, define the affine normal plane $\mathcal{A}(i)$ as the plane generated by $\left\{\phi(i), \phi^{\prime \prime}(i)\right\}$ and the affine focal set $\mathcal{B}=\mathcal{B}(\phi, M)$ as a discrete envelope of these affine normal planes. For silhouette polygons $\phi$ relative to $O$, equation (1.6) reduces to

$$
[\phi(i-1)-O, \phi(i)-O, \phi(i+1)-O]=1,
$$

which is a discrete counterpart of equation 1.2 .

The smooth curves $\phi \subset M$ whose affine focal set $\mathcal{B}$ reduces to a single line were characterized in 4 . Consider a smooth planar curve $\Gamma(t)$ parameterized by affine arc-length and denote by $z(t)$ the affine distance or support function of $\Gamma(t)$ with respect to some point $P \in \mathbb{R}^{2}([1])$. Then the affine focal set of the silhouette curve $\phi(t)=\left(\Gamma^{\prime}(t), z(t)\right)$ reduces to a single line and conversely, if $\mathcal{B}(\phi, M)$ is a single line, then $\phi$ is a silhouette curve obtained by this construction for some planar curve $\Gamma$ and some $P \in \mathbb{R}^{2}$. We prove in this paper a discrete counterpart of this characterization for equal-volume polygons contained in a polyhedron.

Consider a smooth curve $\Phi(t)$ in 3-space parameterized by affine arc-length, i.e., satisfying equation (1.3). The planes through $\Phi$ parallel to $\left\{\Phi^{\prime}, \Phi^{\prime \prime \prime}\right\}$ are called affine rectifying planes and the envelope of the affine rectifying planes $R S(\Phi)$ is called the intrinsic affine binormal developable ([9]). The characterization of curves $\Phi$ such that $R S(\Phi)$ is cylindrical is easily obtained from the characterization of curves $\phi=\Phi^{\prime}$ whose affine focal set is a single line $([4,9])$. A polygon $\Phi\left(i+\frac{1}{2}\right)$ in 3 -space is said to be equal-volume if

$$
\left[\Phi^{\prime}(i-1), \Phi^{\prime}(i), \Phi^{\prime}(i+1)\right]=1,
$$

for all $i$, which is equivalent to say that the difference polygon $\phi(i)=\Phi^{\prime}(i)$ is equal-volume with respect to the origin. Although it is not clear how to obtain a discrete version of the intrinsic affine binormal developable, we can obtain interesting consequences of the discrete characterization of polygons $\phi$ whose affine focal set is a single line.

We can also apply the equal-volume model in a projective setting. Given a smooth planar curve $(\tilde{\phi}(t), 1)$, there exists a projectively equivalent curve $\phi(t)$ in 3-space satisfying equation 1.2 with $O$ equal the origin. From this curve, we can define the projective length $p l(\tilde{\phi})$ (see [7]). For a planar polygon $(\tilde{\phi}(i), 1)$, we can also obtain a projectively equivalent equal-volume polygon $\phi(i)$ in 3 -space and, from this polygon, we obtain two definitions for the projective length, $p l_{1}(\tilde{\phi})$ and $p l_{2}(\tilde{\phi})$, that unfortunately do not coincide. Nevertheless, we prove that if the polygon is obtained from a dense enough 
sampling of a smooth curve, both the discrete projective length $p l_{1}(\tilde{\phi})$ and $p l_{2}(\tilde{\phi})$ are close to the projective length of the smooth curve.

The paper is organized as follows: In section 2 we review the smooth results for affine geometry of curves contained in surfaces, affine geometry of curves in 3-space and projective geometry of planar curves. In section 3 we calculate affine invariants of equal-volume polygons contained in polyhedra. In section 4 , we apply the results of section 3 to compute affine invariants for equalvolume polygons in 3 -space. In section 5 we discuss the projective length of a planar polygon.

\section{Affine geometry of smooth curves in 3-space}

\subsection{Curves contained in surfaces}

Let $\phi: I \rightarrow \mathbb{R}^{3}$ be a curve contained in a surface $M$ and $\xi$ a vector field tangent to $M$ and transversal to $\phi$. We shall assume that $\phi \subset M$ is nondegenerate, i.e., the osculating plane of $\phi$ does not coincide with the tangent plane of $M$ at any point. Under this hypothesis, there exists a vector field $\xi(t)$, unique up to scalar (non-constant) multiple, such that $\xi^{\prime}(t)$ is tangent to $M$, for any $t \in I$. The vector field $\xi$ determines a unique direction tangent to $M$, which is called the Darboux direction along $\phi$. In the Darboux direction, there exists a vector field $\xi(t)$, unique up to a constant multiple, such that $\xi^{\prime}(t)$ is tangent to $\phi(t)$, for any $t \in I$. We call this vector field the parallel Darboux vector field. The parallel Darboux vector field satisfies equation (1.4), for some scalar function $\sigma$.

The envelope of tangent planes is the developable surface

$$
x(t, u)=\phi(t)+u \xi(t) .
$$

This surface is called the Osculating Tangent Developable Surface of $M$ along $\phi$ and will be denoted $\mathcal{E}([3],[10])$. The surface $\mathcal{E}$ is a cone if and only if $\sigma$ is constant. In this case, the vertex of the cone is given by $O=\phi+\sigma^{-1} \xi$ and the curve $\phi$ is a silhouette curve from the point of view of $O$.

Under the non-degeneracy hypothesis, there exists a parameterization $\phi(t)$ of $\phi$, unique up to a translation, such that equation (1.1) holds. The plane $\mathcal{A}(t)$ generated by $\left\{\xi(t), \phi^{\prime \prime}(t)\right\}$ is called the affine normal plane of $\phi \subset M$. Condition 1.1 is equivalent to $\phi^{\prime \prime \prime}(t)$ tangent to $M$. Thus we can write

$$
\phi^{\prime \prime \prime}(t)=-\rho(t) \phi^{\prime}(t)+\tau(t) \xi(t),
$$

for some scalar functions $\rho$ and $\tau$.

There exists a basis $\{\xi(t), \eta(t)\}$ of the affine normal plane $\mathcal{A}(t)$ with $\eta$ parallel, i.e., $\eta^{\prime}$ tangent to $\phi$. In fact, define the vector field $\eta$ by $\eta=\phi^{\prime \prime}+\lambda \xi$, where $\lambda^{\prime}=-\tau$. Taking $\mu=\rho+\lambda \sigma$, we obtain the equation

$$
\eta^{\prime}(t)=-\mu(t) \phi^{\prime}(t)
$$


which in particular says that $\eta$ is parallel. The affine focal set $\mathcal{B}$, or affine evolute, is the envelope of affine normal planes. It is the developable surface generated by the lines passing through $O=\phi+\sigma^{-1} \xi$ and $Q=\phi+\mu^{-1} \eta$. The affine focal set reduces to a single line if and only if $\sigma$ and $\mu$ are constant (4]).

If $\phi$ is contained in a plane $L$, then $\tau(t)=0$ for any $t \in I$ and conversely, if $\tau(t)=0$ for any $t \in I$, then $\phi$ is planar. Denote by $n$ a euclidean unitary normal to $L$ and let $\xi$ be the vector field in the Darboux direction such that $\xi \cdot n=1$, where $\cdot$ denotes the usual inner product. Then $\xi$ is a parallel Darboux vector field. In this case, the adapted parameter $t$ corresponds to the affine arc-length parameter and $\rho(t)$ is the affine curvature of $\phi \subset L$. For planar curves, $\lambda=0$ and so $\phi^{\prime \prime}=\eta \subset L$ is parallel. Then the set $\mathcal{B} \cap L$ coincides with the affine evolute of the planar curve $\phi \subset L([])$.

For silhouette curves, $\xi(t)=\phi(t)-O$ and so equation 1.1 becomes 1.2 , i.e., $\phi(t)$ is parameterized by centro-affine arc-length. Assuming $O$ equals the origin, equation 2.1 becomes

$$
\phi^{\prime \prime \prime}(t)=-\rho(t) \phi^{\prime}(t)+\tau(t) \phi(t) .
$$

Moreover $\mu=\rho-\lambda$, which implies $\mu^{\prime}=\rho^{\prime}+\tau$.

Curves whose affine focal set $\mathcal{B}$ reduces to a single line. The affine focal set reduces to a single line if and only if $\mu$ and $\sigma$ are constant. Since $\sigma$ is constant, $\phi$ is necessarily a silhouette curve from the point of view of $O$, that we shall assume to be the origin. The condition $\mu$ constant can be written as $\rho^{\prime}+\tau=0$. In this case equation 2.3 becomes $\phi^{\prime \prime \prime}=-(\rho \phi)^{\prime}$, which is equivalent to

$$
\phi^{\prime \prime}(t)=-\rho(t) \phi(t)+Q,
$$

for some constant vector $Q$. Assuming that $Q=(0,0,1)$ and writing $\phi(t)=$ $(\gamma(t), z(t))$, this equation becomes

$$
\gamma^{\prime \prime}(t)=-\rho(t) \gamma(t) ; \quad z^{\prime \prime}(t)=-\rho(t) z(t)+1 .
$$

Consider a convex planar curve $\Gamma(t)$. Assume that $\Gamma(t)$ is parameterized by affine arc-length, i.e., $\left[\Gamma^{\prime}(t), \Gamma^{\prime \prime}(t)\right]=1$, and let $\rho(t)$ denotes the affine curvature of $\Gamma$, i.e., $\Gamma^{\prime \prime \prime}(t)=-\rho(t) \Gamma^{\prime}(t), t \in I$. Let $\gamma(t)=\Gamma^{\prime}(t)$ and denote by $z(t)=[\Gamma(t)-P, \gamma(t)]$ the affine distance, or support function, of $\Gamma$ with respect to a point $P \in \mathbb{R}^{2}([1])$.

The following proposition was proved in [4]:

Proposition 2.1. The affine focal set $\mathcal{B}$ of the curve $\phi(t)=(\gamma(t), z(t))$ is a single line, and conversely, any curve $\phi$ whose affine focal set is a single line can be obtained as above, for some planar curve $\Gamma$ and some point $P \in \mathbb{R}^{2}$.

\subsection{Curves in 3-space}

Consider now a curve $\Phi$ in the 3 -space, without being contained in a given surface $M$. We say that a parameterization $\Phi(t)$ of $\Phi$ is by affine arc-length if 
formula 1.3 holds. This condition implies that $\Phi^{\prime \prime \prime \prime}(t)$ belongs to the plane generates by $\phi^{\prime \prime}(t)$ and $\phi^{\prime}(t)$ and thus we obtain equation

$$
\Phi^{\prime \prime \prime \prime}(t)=-\rho(t) \Phi^{\prime \prime}(t)+\tau(t) \Phi^{\prime}(t),
$$

for some scalar functions $\rho$ and $\tau$. Writing $\phi(t)=\Phi^{\prime}(t)$, we observe that equation 2.5 reduces to 2.3 .

The plane passing through $\Phi(t)$ and generated by $\left\{\Phi^{\prime}(t), \Phi^{\prime \prime \prime}(t)\right\}$ is called affine rectifying plane and the envelope $R S(\Phi)$ of the affine rectifying planes is called the intrinsic affine binormal developable of $\Phi$. It is proved in [9] that $R S(\Phi)$ is cylindrical if and only if $\rho^{\prime}+\tau=0$.

Curves with $\mu$ constant. The condition $\mu$ constant is equivalent to $\rho^{\prime}+\tau=0$. Consider a convex planar curve $\Gamma$ parameterized by affine arc-length and let

$$
Z(t)=\int_{t_{0}}^{t}\left[\Gamma(s)-P, \Gamma^{\prime}(s)\right] d s .
$$

Then $Z(t)$ represents the area of the planar region bounded by $\Gamma(s), t_{0} \leq$ $s \leq t$, and the segments $P \Gamma\left(t_{0}\right)$ and $P \Gamma(t)$. The following proposition is a direct consequence of proposition 2.1 .

Proposition 2.2. For the curve $\Phi(t)=(\Gamma(t), Z(t)), \mu$ is constant, and conversely, any curve $\Phi$ in 3-space with $\mu$ constant is obtained by this construction, for some convex planar curve $\Gamma$ and some point $P \in \mathbb{R}^{2}$.

\subsection{Projective invariants}

Consider a parameterized planar curve $\tilde{\phi}(t), t \in I$, without inflection points. Any curve of the form $\phi(t)=a(t) \tilde{\phi}(t)$ is projectively equivalent to $\tilde{\phi}(t)$ and is called a representative of $\tilde{\phi}(t)$. It turns out that there exists a representative $\phi(t)$ of $\tilde{\phi}(t)$ satisfying formula 1.2 with $O$ equal to the origin. Then $\phi^{\prime \prime \prime}(t)$ belongs to the space generated by $\left\{\phi^{\prime}(t), \phi(t)\right\}$ and equation 2.3 holds, for some scalar functions $\rho$ and $\tau$.

The quantity $\rho^{\prime}(t)+2 \tau(t)$ is projectively invariant and $\rho^{\prime}(t)+2 \tau(t)=0$ if and only if $\phi$ is contained in a quadratic cone. In fact,

$$
p l(\tilde{\phi})=\int_{I}\left(\rho^{\prime}(t)+2 \tau(t)\right)^{1 / 3} d t
$$

is the projective length of $\tilde{\phi}$ (see [7]).

\section{Affine geometry of equal-volume polygons contained in polyhedra}

In this section, we obtain discrete counterparts of the results of section 2.1. The derivatives are replaced by differences, and so for a function $f$ : $\{1, \ldots, N\} \rightarrow \mathbb{R}^{k}$, we denote

$$
f^{\prime}\left(i+\frac{1}{2}\right)=f(i+1)-f(i), \quad f^{\prime \prime}(i)=f^{\prime}\left(i+\frac{1}{2}\right)-f^{\prime}\left(i-\frac{1}{2}\right),
$$


and so on.

\subsection{Basic model}

Consider a polyhedron $M$ whose faces are planar quadrilaterals and let $\phi$ be a polygonal line such that each of its sides are connecting opposite edges of a face of $M$. We shall denote by $\phi(i), 1 \leq i \leq N$, the vertices of such polygon and by $\xi(i)$ a vector in the direction of the edges of $M$ containing $\phi(i)$. Edges of $M$ that don't intersect $\phi$ are not important in our model (see Figure 1).

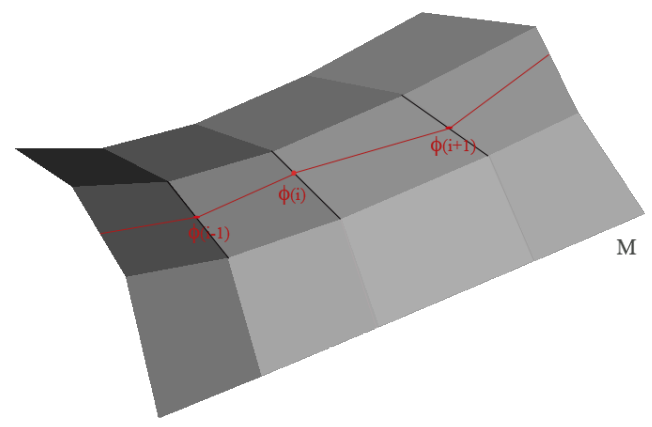

Figure 1. Polygonal line $\phi$ contained in polyhedron $M$.

We shall denote by $T_{i+1 / 2} M$ the face of $M$ that contains the side $i+1 / 2$. By the planar quadrilaterals hypothesis, the vectors $\phi^{\prime}\left(i+\frac{1}{2}\right), \xi(i)$ and $\xi(i+1)$ belong to $T_{i+1 / 2} M$, which is a discrete counterpart of the Darboux condition $\xi^{\prime}$ tangent to $M$. It is clear that there exists $\xi$, unique up to a multiplicative constant, such that $\xi^{\prime}(i+1 / 2)$ is parallel to $\phi^{\prime}(i+1 / 2)$. This vector field is the parallel Darboux vector field of $\phi \subset M$ and equation 1.5 holds, for some scalar function $\sigma$ (see Figure 2).

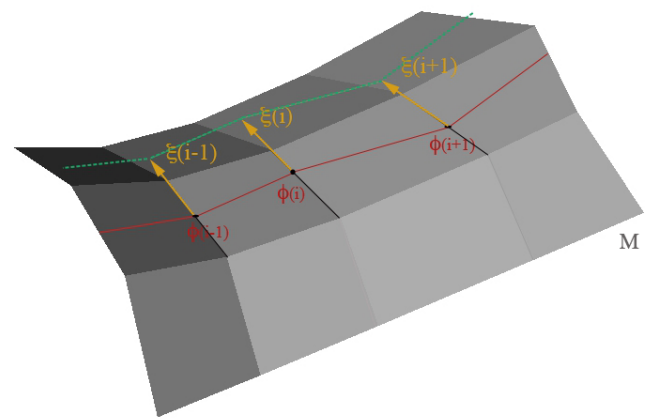

Figure 2. Parallel Darboux vectors field $\xi$. The segments connecting the endpoints of the vectors $\xi(i)$ are parallel to the sides of $\phi$. 


\subsection{Osculating developable polyhedron}

The line $x(i, u)=\phi(i)+u \xi(i), u \in \mathbb{R}$, is the support line of the edge of the polyhedron $M$ that contains $\phi$. Thus $x(i, u)$ and $x(i+1, u)$ are co-planar and denote by $O\left(i+\frac{1}{2}\right)$ the intersection point of these lines. We have that

$$
O\left(i+\frac{1}{2}\right)=\phi(i)+\sigma^{-1}\left(i+\frac{1}{2}\right) \xi(i)=\phi(i+1)+\sigma^{-1}\left(i+\frac{1}{2}\right) \xi(i+1) .
$$

The osculating developable polyhedron $\mathcal{E}$ is the polyhedron whose face $i+1 / 2$ is the region of $T_{i+1 / 2} M$ bounded by $x(i, u)$ and $x(i+1, u)$ and containing the side $i+1 / 2$ of $\phi$ (see Figure 3).

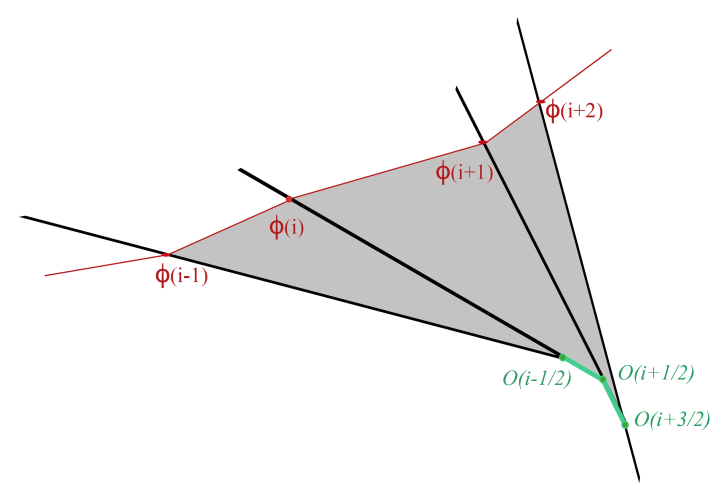

Figure 3. Osculating developable polyhedron $\mathcal{E}$

We have the following proposition:

Proposition 3.1. The following statements are equivalent:

1. $\sigma\left(i+\frac{1}{2}\right)$ does not depend on $i$.

2. The point $O\left(i+\frac{1}{2}\right)$ does not depend on $i$.

3. $\mathcal{E}$ is a cone.

Proof. Observe that

$$
O\left(i+\frac{1}{2}\right)-O\left(i-\frac{1}{2}\right)=\left(\sigma^{-1}\left(i+\frac{1}{2}\right)-\sigma^{-1}\left(i+\frac{1}{2}\right)\right) \xi(i)
$$

Thus (1) is equivalent to (2). The equivalence between (2) and (3) is obvious.

The polygons $\phi \subset M$ for which $\mathcal{E}$ reduces to a point $O$ is the object of study of the centro-affine geometry. In this case, the polygon $\phi$ can be thought as a silhouette polygon of $M$ from the point of view of $O$. 


\subsection{Equal-volume polygons}

We say that the polygon $\phi$ contained in the polyhedron $M$ is equal-volume if equation (1.6) holds.

Lemma 3.2. The polygon $\phi \subset M$ is equal-volume if and only if

$$
\phi^{\prime \prime \prime}\left(i+\frac{1}{2}\right) \in T_{i+1 / 2} M .
$$

Proof. Equation 1.6 is equivalent to

$$
\left[\phi^{\prime}\left(i+\frac{1}{2}\right), \phi^{\prime}\left(i+\frac{3}{2}\right), \xi(i+1)\right]-\left[\phi^{\prime}\left(i-\frac{1}{2}\right), \phi^{\prime}\left(i+\frac{1}{2}\right), \xi(i)\right]=0,
$$

for each $i$, which is equivalent to

$\left[\phi^{\prime}\left(i+\frac{1}{2}\right), \phi^{\prime}\left(i+\frac{3}{2}\right), \xi(i+1)-\xi(i)\right]-\left[\phi^{\prime}\left(i+\frac{1}{2}\right), \phi^{\prime \prime}(i)-\phi^{\prime \prime}(i+1), \xi(i)\right]=0$.

By the parallel Darboux condition, the first parcel is zero and thus the above condition is equivalent to

$$
\left[\phi^{\prime}\left(i+\frac{1}{2}\right), \phi^{\prime \prime}(i)-\phi^{\prime \prime}(i+1), \xi(i)\right]=0,
$$

which is clearly equivalent to $\phi^{\prime \prime \prime}(i+1 / 2)$ belongs to $T_{i+1 / 2} M$.

We shall assume along the paper that the polygon $\phi \subset M$ is equal-volume. By the above lemma we can write

$$
\left\{\begin{array}{l}
\phi^{\prime \prime \prime}\left(i+\frac{1}{2}\right)=-\rho_{2}(i) \phi^{\prime}\left(i+\frac{1}{2}\right)+\tau\left(i+\frac{1}{2}\right) \xi(i+1) \\
\phi^{\prime \prime \prime}\left(i+\frac{1}{2}\right)=-\rho_{1}(i+1) \phi^{\prime}\left(i+\frac{1}{2}\right)+\tau\left(i+\frac{1}{2}\right) \xi(i)
\end{array}\right.
$$

for some scalar functions $\rho_{1}, \rho_{2}$ and $\tau$ satisfying the compatibility equation

$$
-\tau\left(i+\frac{1}{2}\right) \sigma\left(i+\frac{1}{2}\right)=\rho_{2}(i)-\rho_{1}(i+1) .
$$

Equations (3.3) are discrete counterparts of equation (2.1).

Remark 3.3. Starting from a general polygon $\phi \subset M$, we may obtain an equal-volume polygon $\bar{\phi} \subset \bar{M}$ by the following inductive algorithm (see Figure 4):

1. Let $(\bar{\phi}(i), \bar{\xi}(i))=(\phi(i), \xi(i))$, for $i=1,2,3$.

2. Given the pair $(\bar{\phi}, \bar{\xi})$ at $i-1, i$ and $i+1$, consider a plane parallel to $T_{i+1 / 2} \bar{M}$ through $\bar{\phi}(i-1)$ and let $\bar{\phi}(i+2)$ be the intersection of this plane with the polygonal line $\phi$.

3. The direction of the vector $\bar{\xi}(i+2)$ is obtained by linear interpolation of $\xi(k)$ and $\xi(k+1)$, where $k+\frac{1}{2}$ is the index of the side of $\phi$ containing $\bar{\phi}(i+2)$. Thus $\bar{\xi}(i+2) \in T_{k+1 / 2} M$. 


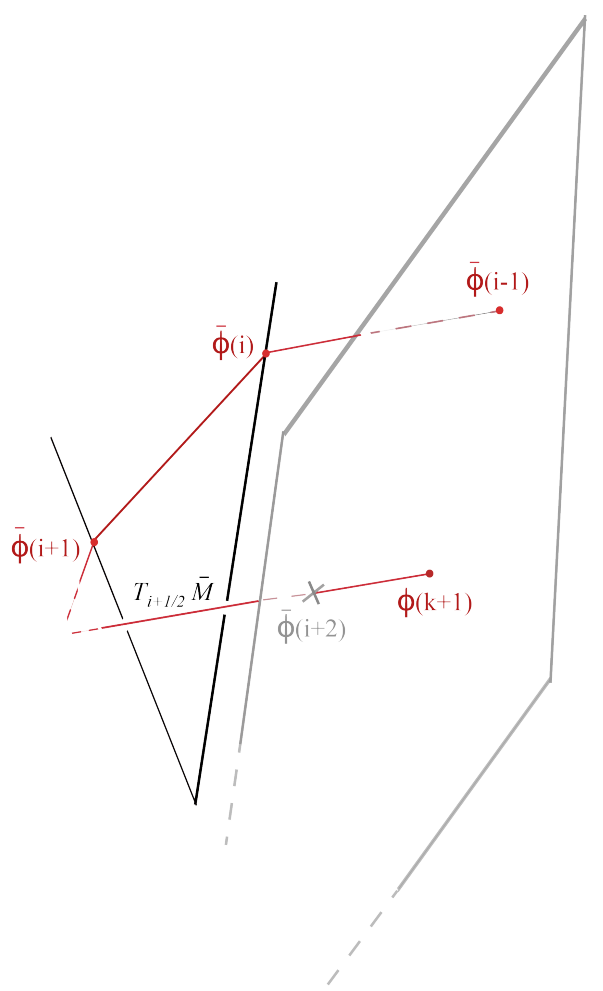

FiguRE 4. Algorithm to construct an equal-volume polygon

\subsection{Discrete affine focal set}

Take any $\lambda$ satisfying $\lambda(i)-\lambda(i+1)=\tau\left(i+\frac{1}{2}\right)$ and define

$$
\eta(i)=\phi^{\prime \prime}(i)+\lambda(i) \xi(i) \text {. }
$$

Define also

$$
\mu\left(i+\frac{1}{2}\right)=\rho_{1}(i+1)+\sigma\left(i+\frac{1}{2}\right) \lambda(i+1)=\rho_{2}(i)+\sigma\left(i+\frac{1}{2}\right) \lambda(i) .
$$

Lemma 3.4. The following discrete counterpart of equation (2.2) holds:

$$
\eta^{\prime}\left(i+\frac{1}{2}\right)=-\mu\left(i+\frac{1}{2}\right) \phi^{\prime}\left(i+\frac{1}{2}\right) .
$$

In particular, $\eta$ is parallel.

Proof. We have that

$$
\begin{gathered}
\eta^{\prime}\left(i+\frac{1}{2}\right)=\phi^{\prime \prime \prime}\left(i+\frac{1}{2}\right)+\lambda^{\prime}\left(i+\frac{1}{2}\right) \xi(i+1)+\lambda(i) \xi^{\prime}\left(i+\frac{1}{2}\right) \\
=-\rho_{2}(i) \phi^{\prime}\left(i+\frac{1}{2}\right)+\lambda(i) \xi^{\prime}\left(i+\frac{1}{2}\right)=-\left(\rho_{2}(i)+\sigma\left(i+\frac{1}{2}\right) \lambda(i)\right) \phi^{\prime}\left(i+\frac{1}{2}\right),
\end{gathered}
$$

thus proving the lemma. 
Define

$$
Q\left(i+\frac{1}{2}\right)=\phi(i)+\mu^{-1}\left(i+\frac{1}{2}\right) \eta(i)=\phi(i+1)+\mu^{-1}\left(i+\frac{1}{2}\right) \eta(i+1),
$$

and denote by $l\left(i+\frac{1}{2}\right)$ the line connecting $O\left(i+\frac{1}{2}\right)$ and $Q\left(i+\frac{1}{2}\right)$, where $O\left(i+\frac{1}{2}\right)$ is defined by equation 3.1 .

The discrete affine focal set $\mathcal{B}$ is the polyhedron with edges $l\left(i+\frac{1}{2}\right), i=$ $1, \ldots, N-1$, and faces contained in $\mathcal{A}(i), i=2, \ldots, N-1$, bounded by $l\left(i-\frac{1}{2}\right)$ and $l\left(i+\frac{1}{2}\right)$ containing the segments $Q\left(i-\frac{1}{2}\right) Q\left(i+\frac{1}{2}\right)$ and $O\left(i-\frac{1}{2}\right) O\left(i+\frac{1}{2}\right)$ (see Figure 5).

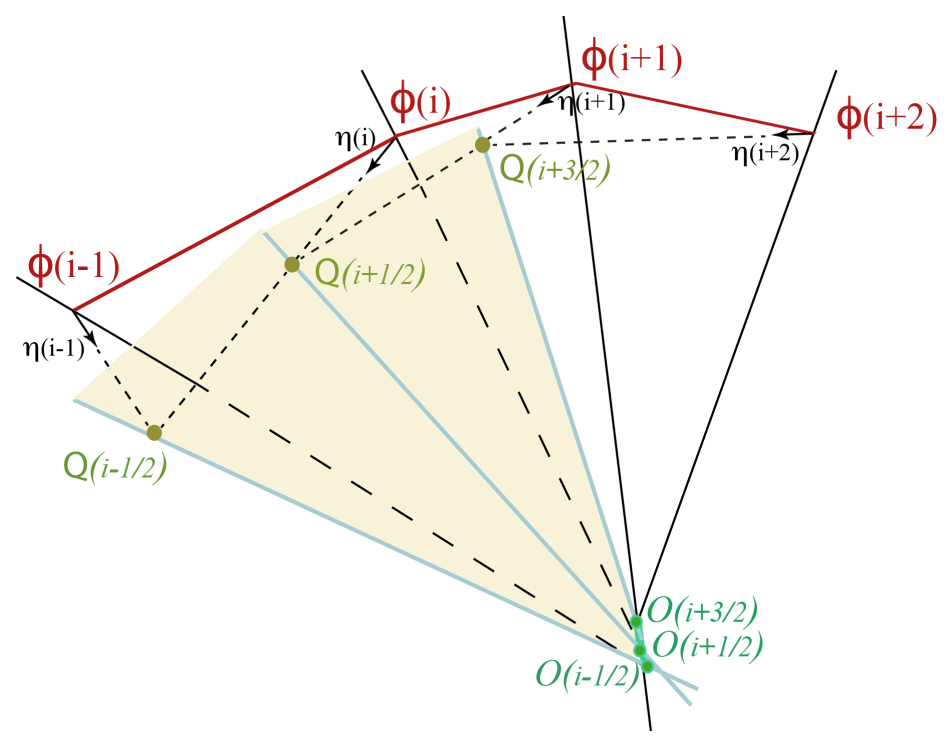

FiguRE 5. Discrete affine focal set

Proposition 3.5. The following statements are equivalent:

1. $\sigma$ and $\mu$ are constant.

2. The points $O$ and $Q$ are fixed.

3. The discrete affine focal set $\mathcal{B}$ reduces to a single line.

Proof. By proposition 3.1 $\sigma$ constant is equivalent to $O$ fixed. From equation (3.8) we obtain

$$
Q\left(i+\frac{1}{2}\right)-Q\left(i-\frac{1}{2}\right)=\left(\mu^{-1}\left(i+\frac{1}{2}\right)-\mu^{-1}\left(i-\frac{1}{2}\right)\right) \eta(i),
$$

which implies that $\mu$ is constant if and only if $Q$ is fixed. Thus (1) and (2) are equivalent. It is obvious that (2) implies (3) and so it remains to prove that (3) implies (2). If $O$ and $Q$ were not both fixed, then equations 3.2 and 3.9 say that $O$ or $Q$ are not changing in the direction of $Q-O$. Thus $\mathcal{B}$ would not be a single line. 


\subsection{Planar polygons}

Lemma 3.6. A polygon $\phi$ is contained in a plane $L$ if and only if $\tau=0$.

Proof. Observe that $\tau\left(i+\frac{1}{2}\right)=0$ if and only if the points $\phi(i-1), \phi(i)$, $\phi(i+1)$ and $\phi(i+2)$ are co-planar.

Denote by $n$ a euclidean unitary normal to $L$ and let $\xi$ be the vector field in the Darboux direction such that $\xi \cdot n=1$, where $\cdot$ denotes the usual inner product. Then $\xi$ is a parallel Darboux vector field. In this case equation (1.6) can be written as

$$
\left[\phi^{\prime}\left(i-\frac{1}{2}\right), \phi^{\prime}\left(i+\frac{1}{2}\right)\right]=1,
$$

where $[\cdot, \cdot]$ denotes determinant in the plane $L$. Thus $\phi \subset L$ is an equal-area polygon and $\rho$ is its discrete affine curvature $([2,[11)$. The set $\mathcal{B} \cap L$ is exactly the discrete affine evolute of the planar equal-area polygon $\phi \subset L$ ([2]) (see Figure 6.

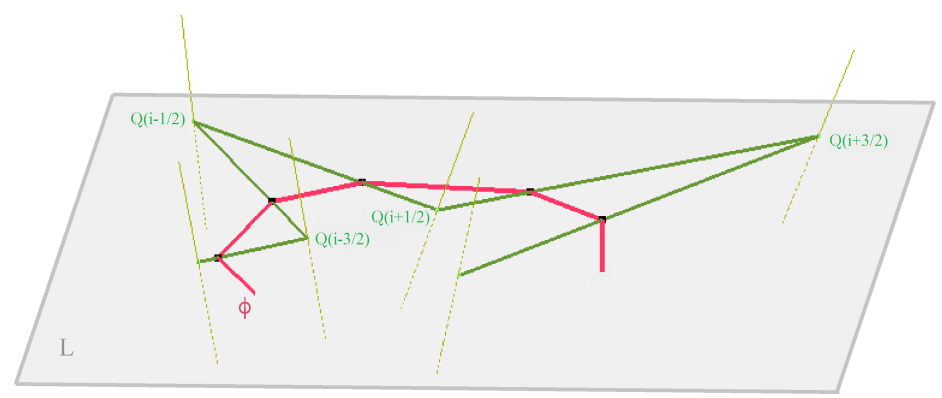

Figure 6. For a planar curve $\phi$, the set $\mathcal{B} \cap L$ coincides with the discrete affine evolute of $\phi$.

\subsection{Silhouette polygons}

Assume that $\phi \subset \mathbb{R}^{3}$ is a silhouette polygon from the point of view of $O$, that we assume to be the origin. In this case, equation $(1.6)$ becomes equation 1.7. By Lemma 3.2 this condition is equivalent to $\phi^{\prime \prime \prime}\left(i+\frac{1}{2}\right)$ belongs to the plane generated by $\phi(i)$ and $\phi(i+1)$.

Since $\xi(i)=\phi(i)$, we have that $\sigma\left(i+\frac{1}{2}\right)=-1, i=1, \ldots, N$. The Frenet equations 3.3 reduce to

$$
\left\{\begin{array}{l}
\phi^{\prime \prime \prime}\left(i+\frac{1}{2}\right)=-\rho_{2}(i) \phi^{\prime}\left(i+\frac{1}{2}\right)+\tau\left(i+\frac{1}{2}\right) \phi(i+1) \\
\phi^{\prime \prime \prime}\left(i+\frac{1}{2}\right)=-\rho_{1}(i+1) \phi^{\prime}\left(i+\frac{1}{2}\right)+\tau\left(i+\frac{1}{2}\right) \phi(i),
\end{array}\right.
$$

while equation $(3.6)$ becomes

$$
\mu\left(i+\frac{1}{2}\right)=\rho_{1}(i+1)-\lambda(i+1)=\rho_{2}(i)-\lambda(i) .
$$


We have also that

$$
\mu^{\prime}(i)=\rho_{1}^{\prime}\left(i+\frac{1}{2}\right)+\tau\left(i+\frac{1}{2}\right)=\rho_{2}^{\prime}\left(i-\frac{1}{2}\right)+\tau\left(i-\frac{1}{2}\right) .
$$

\subsection{Polygons whose discrete affine focal set reduces to a line}

By proposition 3.5, $\mathcal{B}$ reduces to a single line if and only if $\mu$ and $\sigma$ are constant. Since $\sigma$ is constant, $\phi$ is a silhouette polygon. By formula 3.12, the condition $\mu$ constant is equivalent to $\rho_{1}^{\prime}+\tau=\rho_{2}^{\prime}+\tau=0$.

Assume $\mu\left(i+\frac{1}{2}\right)=\mu_{0}$ constant. Then equation (3.7) implies that

$$
\eta(i)=-\mu_{0} \phi(i)+Q
$$

for some constant vector $Q$. Assume $Q=(0,0,1)$ and write $\phi(i)=(\gamma(i), z(i))$. Then, using equation 3.5 we obtain

$$
\gamma^{\prime \prime}(i)+\lambda(i) \gamma(i)=-\mu_{0} \gamma(i), \quad z^{\prime \prime}(i)+\lambda(i) z(i)=-\mu_{0} z(i)+1,
$$

and so

$$
\gamma^{\prime \prime}(i)=-\left(\lambda(i)+\mu_{0}\right) \gamma(i), \quad z^{\prime \prime}(i)=-\left(\lambda(i)+\mu_{0}\right) z(i)+1 .
$$

Observe that

$$
[\gamma(i), \gamma(i+1)]-[\gamma(i-1), \gamma(i)]=\left[\gamma(i), \gamma^{\prime \prime}(i)\right]=0,
$$

and so $[\gamma(i), \gamma(i+1)]=c$, for some constant $c$. By rescaling $\phi$ we may assume that $c=1$.

Denote by $\Gamma\left(i+\frac{1}{2}\right)$ a polygon such that $\Gamma^{\prime}(i)=\gamma(i)$. Then

$$
\Gamma^{\prime \prime \prime}(i)=-\left(\lambda(i)+\mu_{0}\right) \Gamma^{\prime}(i)
$$

and so $\Gamma$ is an equal-area polygon with discrete affine curvature $\lambda(i)+\mu_{0}$. The affine distance or support function of $\Gamma$ with respect to a point $P \in \mathbb{R}^{2}$ is given by

$$
z(i)=\left[\Gamma\left(i+\frac{1}{2}\right)-P, \gamma(i)\right]=\left[\Gamma\left(i-\frac{1}{2}\right)-P, \gamma(i)\right]
$$

(see Figure 7, left).

Proposition 3.7. The polygonal line $\phi(i)=(\gamma(i), z(i))$ (see Figure 7 , right) satisfies equation (3.13), and conversely, any solution of the difference equation (3.13) is obtained by this construction, for some planar polygon $\Gamma\left(i+\frac{1}{2}\right)$ and some point $P \in \mathbb{R}^{2}$.

Proof. Observe first that

Thus

$$
\begin{gathered}
z^{\prime}\left(i+\frac{1}{2}\right)=\left[\Gamma\left(i+\frac{1}{2}\right)-P, \gamma(i+1)\right]-\left[\Gamma\left(i+\frac{1}{2}\right)-P, \gamma(i)\right] \\
=\left[\Gamma\left(i+\frac{1}{2}\right)-P, \gamma^{\prime}\left(i+\frac{1}{2}\right)\right] .
\end{gathered}
$$

$$
\begin{gathered}
z^{\prime \prime}(i)=\left[\Gamma\left(i+\frac{1}{2}\right)-P, \gamma^{\prime}\left(i+\frac{1}{2}\right)\right]-\left[\Gamma\left(i-\frac{1}{2}\right)-P, \gamma^{\prime}\left(i-\frac{1}{2}\right)\right] \\
=\left[\gamma(i), \gamma^{\prime}\left(i+\frac{1}{2}\right)\right]+\left[\Gamma\left(i-\frac{1}{2}\right)-P, \gamma^{\prime \prime}(i)\right] \\
=1-\left(\lambda(i)+\mu_{0}\right) z(i)
\end{gathered}
$$



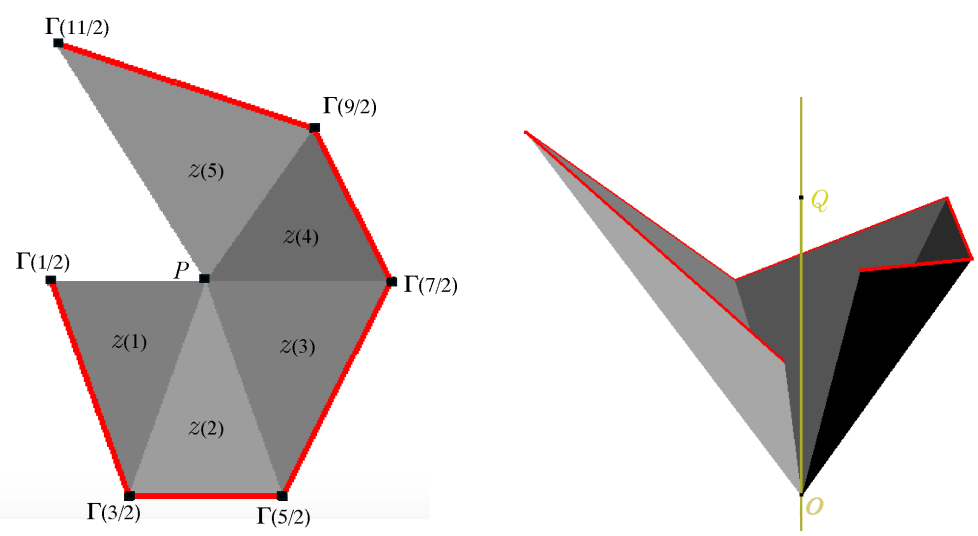

Figure 7. A planar equal-area polygon $\Gamma$ and its support function $z$ (left). The corresponding spatial curve $\phi$ and its affine focal set (right).

thus proving that $(\phi(i), z(i))$ satisfies equation 3.13 . Since $P$ has two degrees of freedom, this is the general solution of the second order difference equation 3.13 .

\section{Polygons in 3-space}

In this section, we obtain discrete counterparts of the results of section 2.2 . Consider a polygon $\Phi\left(i+\frac{1}{2}\right)$ in 3-space, without being contained in any polyhedron $M$. The polygon $\Phi$ is equal-volume, i.e., satisfies equation (1.8), if and only if the difference polygon $\phi(i)=\Phi^{\prime}(i)$ is equal-volume with respect to the origin.

\subsection{Frenet equations}

For equal-volume polygons $\Phi$, Frenet equations 3.10 are written as

$$
\left\{\begin{array}{l}
\Phi^{\prime \prime \prime \prime}\left(i+\frac{1}{2}\right)=-\rho_{2}(i) \Phi^{\prime \prime}\left(i+\frac{1}{2}\right)+\tau\left(i+\frac{1}{2}\right) \Phi^{\prime}(i+1) \\
\Phi^{\prime \prime \prime \prime}\left(i+\frac{1}{2}\right)=-\rho_{1}(i+1) \Phi^{\prime \prime}\left(i+\frac{1}{2}\right)+\tau\left(i+\frac{1}{2}\right) \Phi^{\prime}(i) .
\end{array}\right.
$$

Defining $\mu\left(i+\frac{1}{2}\right)$ by equation 3.11, equation 3.12 still holds. It is not clear how to define a discrete version of the intrinsic affine binormal developable.

\subsection{Polygons with $\mu$ constant}

Consider an equal area planar polygon $\Gamma\left(i+\frac{1}{2}\right)$ and let $Z\left(i+\frac{1}{2}\right)$ be given by

$$
Z\left(i+\frac{1}{2}\right)=\sum_{j=1}^{i} z(j)
$$


where $z(i)$ is given by equation 3.14 , for some point $P \in \mathbb{R}^{2}$. Then $Z\left(i+\frac{1}{2}\right)$ represents the area of the planar region bounded by $\Gamma^{\prime}(j), j=1 \ldots i$, and the segments $P \Gamma\left(\frac{1}{2}\right)$ and $P \Gamma\left(i+\frac{1}{2}\right)$ (see Figure 8). In this context, Proposition 3.7 can be written as follows:

Proposition 4.1. The polygon $\Phi=(\Gamma, Z)$ has constant $\mu$, and conversely, any equal-volume polygon $\Phi$ with constant $\mu$ is obtained by this construction, for some planar polygonal line $\Gamma$ and some point $P \in \mathbb{R}^{2}$.

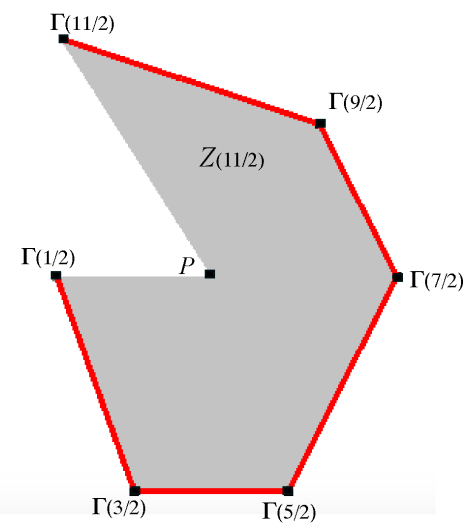

Figure 8. A planar equal-area polygon $\Gamma$ and the area represented by $Z\left(\frac{11}{2}\right)$.

\section{Projective polygons}

In this section, we obtain discrete counterparts of the results of section 2.3 Consider a planar polygon $\tilde{\phi}(i), i=1, \ldots, N$. Assume that

$$
\left[\tilde{\phi}^{\prime}\left(i-\frac{1}{2}\right), \tilde{\phi}^{\prime}\left(i+\frac{1}{2}\right)\right]=b(i)>0 \text {. }
$$

\subsection{Equal-volume representative}

Any polygon $\phi$ in $\mathbb{R}^{3}$ of the form $\phi(i)=a(i)(\tilde{\phi}(i), 1), a(i)>0$, is a projective representative of $\tilde{\phi}$.

Lemma 5.1. There exists a projective representative $\phi$ of $\tilde{\phi}$ such that equation (1.7) holds with $O$ equal to the origin.

Proof. Observe first that

$$
[\phi(i-1), \phi(i), \phi(i+1)]=a(i-1) a(i) a(i+1)\left[\tilde{\phi}^{\prime}\left(i-\frac{1}{2}\right), \tilde{\phi}^{\prime}\left(i+\frac{1}{2}\right)\right] .
$$


So we need to choose $a(i), i=1 \ldots, N$ such that

$$
a(i-1) a(i) a(i+1) b(i)=c, \quad i=2, \ldots, N-1,
$$

for some constant $c$. Since by the hypothesis $(5.1) b(i)>0$, given $a(1)>0$ and $a(2)>0$ we can find unique $a(i)>0, i=3, \ldots, N$ such that 5.2 holds.

Assume that $\phi$ is a representative of $\tilde{\phi}$ such that equation 1.7 holds with $O$ equal to the origin (Figure 9). Then, by lemma 3.2 $\phi^{\prime \prime \prime}\left(i+\frac{1}{2}\right)$ belongs to the plane generated by $\{\phi(i), \phi(i+1)\}$. So we can use equations 3.10 to define $\rho_{1}(i), \rho_{2}(i)$ and $\tau\left(i+\frac{1}{2}\right)$.
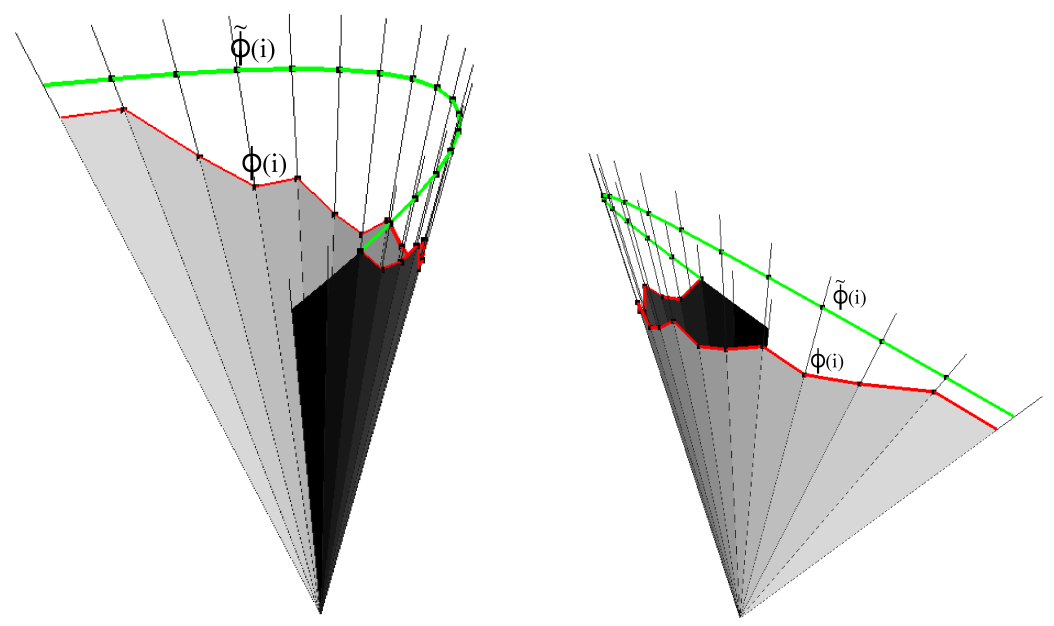

FiguRE 9. Two views of a planar projective polygon $\tilde{\phi}$ and its equal-volume representative $\phi$.

\subsection{Projective length}

We would like to define the projective length of $\tilde{\phi}$ as

$$
p l_{1}(\tilde{\phi})=\sum_{j=2}^{N-2}\left(\rho_{1}^{\prime}\left(i+\frac{1}{2}\right)+2 \tau\left(i+\frac{1}{2}\right)\right)^{1 / 3}
$$

or

$$
p l_{2}(\tilde{\phi})=\sum_{j=2}^{N-2}\left(\rho_{2}^{\prime}\left(i+\frac{1}{2}\right)+2 \tau\left(i+\frac{1}{2}\right)\right)^{1 / 3}
$$

but unfortunately these two definitions do not coincide. Nevertheless, if the polygonal line is obtained from a dense enough sampling of a smooth curve, both of these formulas are close to projective length of the smooth curve given by equation (2.6). Denote by $O\left(h^{k}\right)$ any quantity such that $\lim _{h \rightarrow 0} \frac{O\left(h^{k}\right)}{h^{k-\epsilon}}=0$, for any $\epsilon>0$. 
Lemma 5.2. Assume that the polygonal line $\phi(i), i=1, \ldots N$, is obtained from $\phi(t), 0 \leq t \leq T$, by uniform sampling. Then, for $N h=T$, ih $=t$, we have

$$
\rho_{1}^{\prime}\left(i+\frac{1}{2}\right)+2 \tau\left(i+\frac{1}{2}\right)=\left(\rho^{\prime}(t)+2 \tau(t)\right) h^{3}+O\left(h^{4}\right) .
$$

A similar result holds for $\rho_{2}$.

Proof. It is standard in numerical analysis that $\phi^{\prime}\left(i+\frac{1}{2}\right)=h \phi^{\prime}(t)+O\left(h^{2}\right)$ and $\phi^{\prime \prime \prime}\left(i+\frac{1}{2}\right)=\phi^{\prime \prime \prime}(t) h^{3}+O\left(h^{4}\right)$. Thus equation 2.3 can be written as

$$
\phi^{\prime \prime \prime}\left(i+\frac{1}{2}\right)=-\rho(t) h^{2} \phi^{\prime}\left(i+\frac{1}{2}\right)+\tau(t) h^{3} \phi(i)+O\left(h^{4}\right) .
$$

We conclude that $\tau\left(i+\frac{1}{2}\right)=\tau(t) h^{3}+O\left(h^{4}\right)$ and $\rho_{1}(i+1)=\rho(t) h^{2}+c(t) h^{3}+$ $O\left(h^{4}\right)$. This last equation implies that $\rho_{1}^{\prime}\left(i+\frac{1}{2}\right)=h^{3} \rho^{\prime}(t)+O\left(h^{4}\right)$. Thus we conclude that

$$
\rho_{1}^{\prime}\left(i+\frac{1}{2}\right)+2 \tau\left(i+\frac{1}{2}\right)=\left(\rho^{\prime}(t)+2 \tau(t)\right) h^{3}+O\left(h^{4}\right),
$$

which proves the lemma.

From this lemma we can obtain the following convergence result:

Corollary 5.3. The discrete projective lengths given by equations (5.3) and (5.4) converge to the smooth projective length given by (2.6) when $h \rightarrow 0$.

Example 1. Consider

$$
\tilde{\phi}(t)=(\exp (-t) \cos (t), \exp (-t) \sin (t), 1), \quad 0 \leq t \leq 2 \pi .
$$

Then $\tilde{\phi}$ is projectively equivalent to $\phi(t)=2^{-1 / 3} \exp (2 t / 3) \tilde{\phi}(t)$, which satisfies equation $\sqrt{1.2}$ with $O$ equal the origin. Straightforward calculations show that $\rho(t)=2 / 3, \tau(t)=20 / 27$ and

$$
\operatorname{pl}(\tilde{\phi})=2 \pi \frac{\sqrt[3]{40}}{3} \approx 7.162519249 .
$$

We have done some experiments considering uniform samplings of this curve with $N$ points. Table 1 presents the results for $N=10,100,1000$. Observe that both $p l_{1}(\tilde{\phi})$ and $p l_{2}(\tilde{\phi})$ get closer to $p l(\tilde{\phi})$ as $h=\frac{2 \pi}{N}$ decreases.

TABLE 1. Experimental results of example 1.

\begin{tabular}{cccc}
$\mathrm{N}$ & $\mathrm{h}$ & $p l_{1}(\tilde{\phi})$ & $p l_{2}(\tilde{\phi})$ \\
\hline \hline 10 & 0.62831 & 4.26627 & 3.55522 \\
\hline 100 & 0.06283 & 6.87572 & 6.80410 \\
\hline 1000 & 0.00628 & 7.13407 & 7.12691 \\
\hline
\end{tabular}




\title{
References
}

[1] T. Cecil, Focal points and support functions in affine differential geometry, Geom. Dedicata, 50, 291-300, 1994.

[2] M. Craizer, R. C. Teixeira and M. A. H. B. da Silva: Affine properties of convex equal-area polygons, Disc.Comp.Geom. 48(3), 580-595, 2012.

[3] M. Craizer, M. J. Saia and L. F. Sánchez: Equiaffine Darboux frames for codimension 2 submanifolds contained in hypersurfaces, to appear in J.Math.Soc.Japan, 2016.

[4] M. Craizer, M. J. Saia and L. F. Sánchez: Affine focal sets of codimension 2 submanifolds contained in hypersurfaces, pre-print, 2016.

[5] D. Davis: Generic affine differential geometry of curves in $\mathbb{R}^{n}$, Proc. Royal. Soc. Edinburgh: Sec.A. Math., 136(6), 1195-1205, 2006.

[6] P. J. Giblin and T. Sano: Generic equi-centro-affine differential geometry of plane curves, Topology and its Appl., 159, 476-483, 2012.

[7] L. Guieu, E. Mourre and V. Yu. Ovsienko: Theorem on six vertices of a plane curve via the Sturm theory, The Arnold-Gelfand Math.Seminars (Geom.Sing.Theory), Birkhäuser, Boston, 1997.

[8] S. Izumiya and T. Sano, Affine differential geometry of plane curves and singularity theory, Proc. Royal. Soc. Edinburgh: Sec.A. Math., 128A, 301-314, 1998.

[9] S. Izumiya and T. Sano, Affine differential geometry of space curves and singularity theory, Proc. Edinburgh. Math. Soc., 41, 315-324, 1998.

[10] S. Izumiya and S. Otani, Flat approximations of surfaces along curves, Demonst.Math., 48(2), 217-241, 2015.

[11] F. Käferböck: Affine arc-length polylines and curvature continuous uniform Bsplines, Comp.Aid.Geom.Design, 31(7-8), 331-344, 2014.

\author{
Marcos Craizer \\ Departamento de Matemática- Pontifícia Universidade Católica do Rio de Janeiro \\ Rio de Janeiro-RJ- Brazil \\ e-mail: craizer@puc-rio.br \\ Sinesio Pesco \\ Departamento de Matemática- Pontifícia Universidade Católica do Rio de Janeiro \\ Rio de Janeiro-RJ- Brazil \\ e-mail: sinesio@puc-rio.br
}

